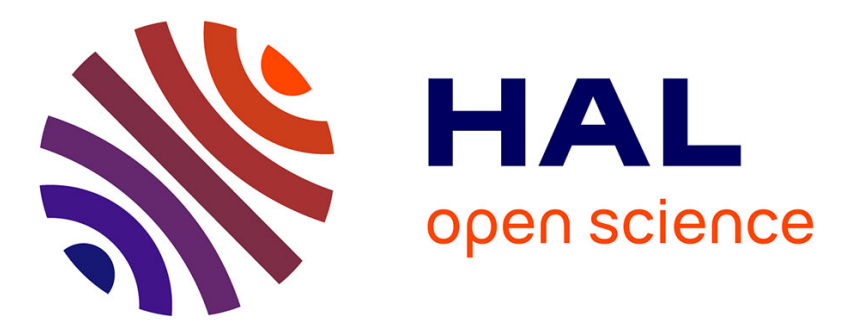

\title{
Etude des propriétés optiques et de la structure de couches minces électrochromes de WO3 préparées par CVD
}

D. Davazoglou, A. Donnadieu, R. Fourcade, Anne Hugot-Legoff, P. Delichère, A. Perez

\section{To cite this version:}

D. Davazoglou, A. Donnadieu, R. Fourcade, Anne Hugot-Legoff, P. Delichère, et al.. Etude des propriétés optiques et de la structure de couches minces électrochromes de WO3 préparées par CVD. Revue de Physique Appliquée, 1988, 23 (3), pp.265-272. 10.1051/rphysap:01988002303026500 . jpa00245770

\section{HAL Id: jpa-00245770 https://hal.science/jpa-00245770}

Submitted on 1 Jan 1988

HAL is a multi-disciplinary open access archive for the deposit and dissemination of scientific research documents, whether they are published or not. The documents may come from teaching and research institutions in France or abroad, or from public or private research centers.
L'archive ouverte pluridisciplinaire HAL, est destinée au dépôt et à la diffusion de documents scientifiques de niveau recherche, publiés ou non, émanant des établissements d'enseignement et de recherche français ou étrangers, des laboratoires publics ou privés. 
Classification

Physics Abstracts

$61.10-78.30 \mathrm{~J}-78.65 \mathrm{~S}-78.90$

\title{
Etude des propriétés optiques et de la structure de couches minces électrochromes de $\mathrm{WO}_{3}$ préparées par CVD
}

\author{
D. Davazoglou $\left({ }^{*, 1}\right)$, A. Donnadieu $\left({ }^{*, 1}\right)$, R. Fourcade $(* *, 1)$, A. Hugot-Legoff $\left({ }^{2}\right)$, \\ P. Delichère $\left({ }^{2}\right)$ et $A$. Perez $\left({ }^{3}\right)$ \\ (*) Laboratoire de Spectroscopie II, Unité Associée au CNRS n 790 \\ (**) Laboratoire des Acides Minéraux, U.A. 79 \\ (1) Université des Sciences et Techniques du Languedoc, place Eugène-Bataillon, 34060 Montpellier Cedex, \\ France \\ (2) Laboratoire de Physique des Liquides et Electrochimie, Groupe de Recherche $n^{\circ} 4$ du CNRS, Université \\ P. et M. Curie, 4 place Jussieu, 75230 Paris, France \\ (3) Département de Physique des Matériaux, Associé au CNRS, Université Claude Bernard Lyon I, 43 bd du \\ 11-Novembre-1918, 69622 Villeurbanne, France
}

(Reçu le 9 septembre 1987, révisé le 23 novembre 1987, accepté le 16 décembre 1987)

\begin{abstract}
Résumé. - Des couches minces polycristallines de $\mathrm{WO}_{3}$ ont été produites par la décomposition en phase vapeur (CVD) de l'hexacarbonyle de tungstène suivie de recuit à température variable en atmosphère oxydante. Les échantillons obtenus en fonction de différentes conditions de préparation ont été caractérisés par diffraction de RX, spectroscopie Raman et rétrodiffusion Rutherford de particules alpha. Leurs caractéristiques optiques sont déterminées à partir des spectres de réflexion et transmission. Tous les échantillons obtenus présentent l'effet électrochrome en présence d'un électrolyte acide.
\end{abstract}

\begin{abstract}
WO}_{3}$ polycrystalline thin films were produced by chemical vapour decomposition (CVD) of tungsten hexacarbonyl followed by annealing at various temperatures and in different annealing environments. Samples thus obtained were characterized by X-ray diffraction, Raman spectroscopy and Rutherford backscattering of alpha particles. Their optical properties were determined from reflection and transmission spectra. All prepared samples exhibit an reversible electrochromic effect in presence of an acid electrolyte.
\end{abstract}

\section{Introduction.}

La découverte vers les années 70 du phénomène électrochrome au sein des couches minces de $\mathrm{WO}_{3}$ [1], a créé un grand intérêt pour l'étude de ce matériau sous forme de couches minces plus ou moins désordonnées. Le phénomène électrochrome est la particularité de certains matériaux, organiques ou minéraux, de changer de coloration de manière réversible lors du passage au travers de leur volume d'un courant, ayant une origine électronique et/ou ionique [2]. Les applications possibles sont d'une part l'affichage électro-optique [3-6] et d'autre part le transfert d'énergie rayonnante [7-11]. Dans les deux cas le but final est l'obtention du contrôle, de manière réversible, des propriétés optiques d'un dispositif d'affichage ou d'un vitrage, afin de pouvoir visualiser l'information ou réguler l'énergie lumineuse au travers d'une fenêtre. Plusieurs types de matériaux fabriqués de diverses manières ont été caractérisés et testés face à certains paramètres dans un but de commercialisation du dispositif électrochrome. Dans le cas des vitrages les paramètres à considérer sont, entre autres, la longue durée de vie, la température de fonctionnement, la résistance aux produits chimiques de nettoyage, etc. Dans ce travail nous présentons des résultats concernant la préparation et la caractérisation de couches minces de $\mathrm{WO}_{3}$. Ces couches sont obtenues par recuits, de différentes façons, de films de «tungstène noir » 
(WN) et de "tungstène réflecteur» (WR), ces dernières préparées par décomposition en phase vapeur (CVD) de l'hexacarbonyle de tungstène. La composition des matériaux est déterminée par mesures de rétrodiffusion Rutherford de particules alpha (RBS) et la structure par diffraction de rayons $\mathrm{X}$ et spectroscopie Raman. Les caractéristiques optiques (indices de réfraction et d'extinction) sont déduites de spectres de réflexion et transmission. Toutes ces mesures de caractérisation sont faites sur les échantillons de $\mathrm{WO}_{3}$ fraichement préparés (donc anhydres) déposés sur supports appropriés (carbone, quartz). Ce type de couches minces déposées sur support de pyrex déjà recouvert d'une couche de $\mathrm{SnO}_{2}$ forme l'électrode de travail d'une cellule électrochimique, contenant, comme électrolyte, une solution normale de $\mathrm{H}_{2} \mathrm{SO}_{4}$, la deuxième électrode étant constituée d'un disque de platine. Cette électrode de travail présente un effet électrochrome réversible suivant le sens de la polarisation. Aucune mesure de caractérisation (RX, Raman ou RBS) n'a été effectuée sur ces électrodes de travail, c'est-à-dire sur les couches de $\mathrm{WO}_{3}$ (colorées ou non) après avoir été mises en contact avec l'électrolyte aqueux.

\section{Processus expérimental.}

2.1 Préparation. - Les détails concernant la préparation des couches de WN ou WR sur support de quartz sont donnés dans la référence [12]. On rappelle cependant que le dépôt se fait dans une enceinte en quartz, par pyrolyse de $\mathrm{W}(\mathrm{CO})_{6}$, à la température de $400^{\circ} \mathrm{C}$ en présence ou non d'un courant d'oxygène suivant le composé recherché : WN ou WR respectivement. Après leur obtention les couches subissent trois types de recuits thermiques $[13,14]$ : à $600^{\circ} \mathrm{C}$ ou à $500^{\circ} \mathrm{C}$ dans l'air (mode 1 ou mode 2 ) ou à $500^{\circ} \mathrm{C}$ dans l'air enrichi en oxygène par un courant d'un mélange $90 \% \mathrm{Ar}-10 \%$ $\mathrm{O}_{2}$ dont le débit est de $1,5 \mathrm{cc} / \mathrm{min}$ (mode 3 ). Tous les recuits dans l'air ou dans l'atmosphère enrichie en oxygène sont réalisés dans la même enceinte que celle utilisée pour la pyrolyse. La température de recuit est atteinte après $20 \mathrm{~min}$ environ, alors commence la mesure du temps d'oxydation (de même que l'introduction de l'oxygène, mode 3) qui se termine quand l'échantillon devient transparent. Pendant le traitement thermique, l'épaisseur de la couche augmente. On définit le rapport des épaisseurs, après et avant recuit, comme le coefficient de gonflement $G$, dont la valeur dépend des conditions d'oxydation et de la nature de la couche initiale (WN ou WR). Pour obtenir une configuration électrochrome, on dépose la couche de $\mathrm{WO}_{3}$, par un des modes déjà décrits, sur support de pyrex préalablement recouvert d'une couche de $\mathrm{SnO}_{2}$, conducteur transparent [15].
2.2 Diffraction DE RAYONS X. - Les mesures de diffraction de rayons $X$ ont été effectuées en utilisant un diffractomètre Philips. La radiation monochromatique utilisée est celle émise par la raie $\mathrm{K}_{\alpha}$ d'une cible de cuivre $(\lambda=0,15405 \mathrm{~nm})$. Le courant, à travers le tube de production des rayons $X$, était $20 \mathrm{~mA}$ et la puissance $800 \mathrm{~W}$.

La faible capacité à la réflexion de couches minces non orientées de $\mathrm{WO}_{3}$ empêche la détermination précise de la position des raies, l'enregistrement devant être effectué dans des conditions extrêmes qui s'accompagnent d'un bruit de fond important.

Les mesures ont dès lors été effectuées à l'aide d'un prototype expérimental permettant l'accumulation des spectres par balayages successifs puis traitement du signal. Le bruit de fond est ainsi très diminué et l'exploitation des spectres est envisageable après une série de 40 accumulations.

2.3 Spectroscopie RAMAN. - Les spectres Raman ont été obtenus au moyen d'un appareil DILOR OMARS 89 muni d'une détection multicanal. L'éclairement de la surface, comme la collecte de la lumière Raman diffusée s'effectuent par l'intermédiaire d'un microscope. On utilise la raie $514,5 \mathrm{~nm}$ d'un laser à Argon; compte tenu de la forte section efficace de diffusion des oxydes de tungstène, de bons spectres sont obtenus en quelques minutes avec une puissance lumineuse de $400 \mathrm{~mW}$ environ.

2.4 RÉTRODIFFUSION DE RUTHERFORD (R.B.S.). Les mesures de rétrodiffusion Rutherford de particules alpha d'énergie $2 \mathrm{MeV}$ ont été effectuées au moyen d'un accélérateur de type Van de Graaff. Dans ce cas, les couches étaient déposées sur un substrat léger (carbone) commode pour ce type de mesure.

2.5 Mesures optiques. - Les mesures de réflexion et transmission ont été effectuées dans un domaine spectral allant de 0,25 à $2,5 \mu \mathrm{m}$, au moyen d'un spectromètre Beckman «UV 5240 » à double faisceau muni d'une sphère intégrante (ACTA UV 5200). C'est à partir des spectres obtenus que les caractéristiques optiques (indices, gap optique) de nos couches ont été déterminées.

2.6 Cellule Électrochimique. - La cellule électrochimique utilisée pour montrer l'effet électrochrome est une cellule à deux électrodes, l'une, électrode de travail, formée par la configuration électrochrome décrite précédemment, l'autre, dite contre-électrode, constituée d'un disque de platine. Ces deux électrodes baignent dans une solution normale aqueuse d'acide sulfurique mise dans une cuve à faces parallèles. L'électrode de travail est maintenue parallèle aux parois de la cuve par un système de deux rails en plastique fixés sur le 
couvercle. Le spectre de transmission de l'électrode de travail est obtenu à l'aide du spectromètre Beckman UV 5240. Une deuxième cuve identique à la première et contenant uniquement l'électrolyte est placée sur le deuxième faisceau, ou faisceau de référence, afin d'éliminer l'influence de l'électrolyte et de la cuve.

En appliquant une tension négative sur la couche de $\mathrm{SnO}_{2}$ par rapport à la contre-électrode, les protons provenant de l'électrolyte et les électrons amenés par le conducteur transparent pénètrent dans la couche de $\mathrm{WO}_{3}$ et provoquent sa coloration selon la réaction globale suivante :

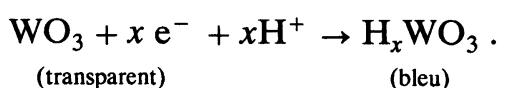

En inversant la polarisation de la tension appliquée, la couche de $\mathrm{WO}_{3}$ se décolore, la réaction étant réversible.

\section{Résultats expérimentaux et discussion.}

3.1 Préparation. - Nos premiers essais ont montré qu'en dessous de $350^{\circ} \mathrm{C}$ l'oxydation n'était pas perceptible. A $400^{\circ} \mathrm{C}$ l'oxydation était très lente ( 2 jours pour une couche de WR de $1000 \AA \AA$ d'épaisseur). A cause de ces temps très longs, nous avons choisi 500 et $600^{\circ} \mathrm{C}$ car à partir de $500^{\circ} \mathrm{C}$ l'oxydation est visible dans des temps relativement courts.

La figure 1 montre les variations du temps d'oxydation avec l'épaisseur de couches de tungstène

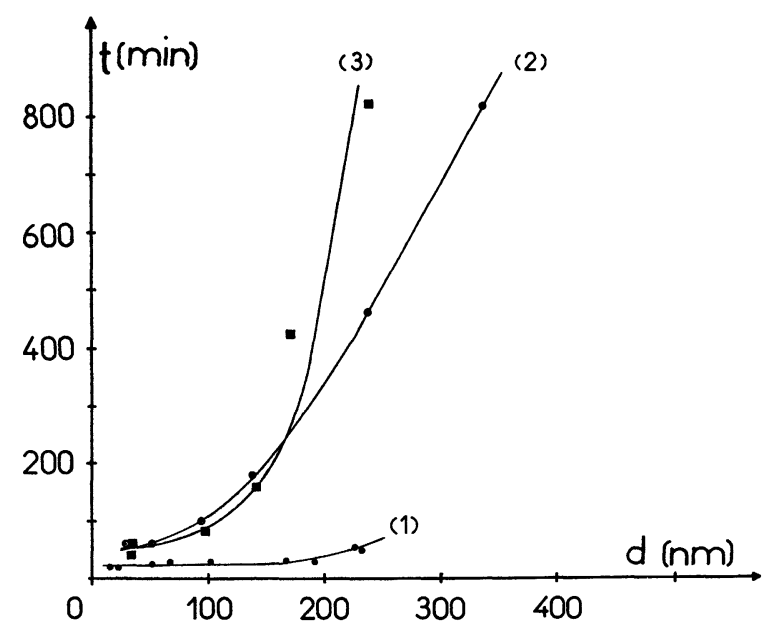

Fig. 1. - Variations du temps d'oxydation en fonction de l'épaisseur de couches minces de tungstène réflecteur (courbe 1 : recuits à $600^{\circ} \mathrm{C}$ dans l'air, courbe 2 : recuits à $500^{\circ} \mathrm{C}$ dans l'air, courbe 3 : recuits à $500^{\circ} \mathrm{C}$ dans l'oxygène).

[Variation of the oxidation time versus thickness of reflective tungsten films (curve 1 : annealed at $600{ }^{\circ} \mathrm{C}$ in air, curve 2 : annealed at $500^{\circ} \mathrm{C}$ in air, curve 3 : annealed at $500{ }^{\circ} \mathrm{C}$ in oxygen).] réflecteur. Les courbes 1,2 et 3 correspondent aux différents modes de recuits utilisés.

Les résultats, très semblables, concernant les couches de tungstène noir (WN) ont été publiés [13].

On observe une nette différence dans la forme des courbes obtenues à $500^{\circ} \mathrm{C}$ et à $600^{\circ} \mathrm{C}$. A température plus élevée les temps de recuit sont nettement plus courts. L'effet du flux d'oxygène, lors du recuit, influence aussi, à partir d'une certaine épaisseur de la couche initiale, la forme de la courbe en augmentant le temps nécessaire à l'oxydation complète. Ceci pourrait être dû à la formation en surface d'une couche mieux cristallisée et plus imperméable à la diffusion des atomes d'oxygène.

Les variations du temps d'oxydation avec l'épaisseur montrent cependant une allure en bon accord avec celle décrite dans la littérature [17-18] pour les mêmes domaines de température d'oxydation.

\subsection{STRUCTURE DES FILMS.}

3.2.1 Analyse aux RX. - La figure 2 représente le spectre de diffraction de rayons $\mathrm{X}$ obtenu par accumulation sur un échantillon $\mathrm{WN}$ recuit à $600^{\circ} \mathrm{C}$ dans l'air. Le spectre est semblable pour tous nos échantil-

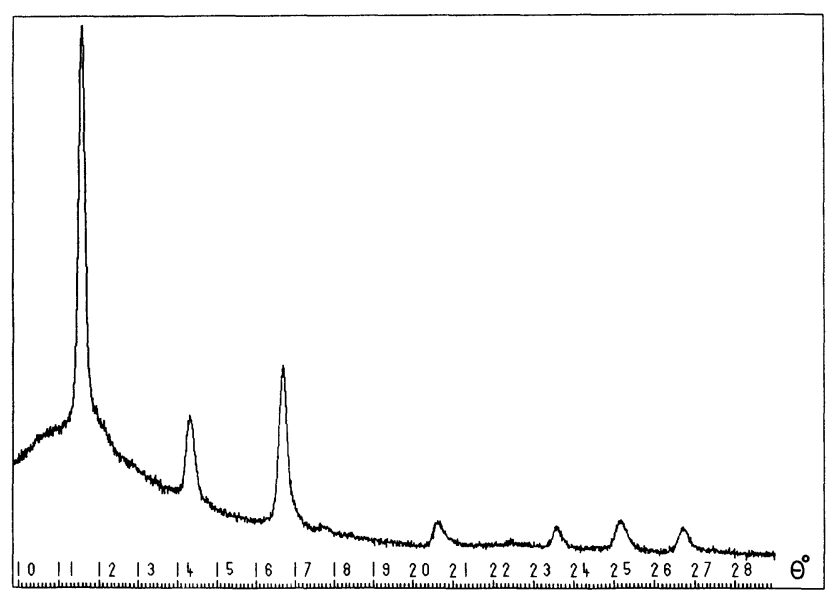

Fig. 2. - Spectre de rayons $X$, sur un échantillon WN recuit à $600^{\circ} \mathrm{C}$ dans l'air.

[XRD spectra taken on a WN sample annealed at $600^{\circ} \mathrm{C}$ in air.]

lons et correspond à la modification monoclinique $\mathrm{du} \mathrm{WO}_{3}$ [19-20]. Les légers décalages observés, tableau I, concernant la position de certaines raies peuvent être dus d'une part au fait que nos couches sont polycristallines et non parfaitement orientées et d'autre part, à la présence d'impuretés : le W $(\mathrm{CO})_{6}$ initial de chez Merck est fourni avec une pureté de $98 \%$.

Un travail beaucoup plus précis tenant compte, pour chaque couche, d'un affinement de paramètres cristallins en fonction de la déconvolution des spectres par profil de raies est actuellement en cours. Ce 
Tableau I. - Position des pics de diffraction de RX. [Positions of the XRD peaks.]

\begin{tabular}{cccccccccc}
\hline $\begin{array}{c}\theta^{\circ} \\
\text { Références }\end{array}$ & $\begin{array}{c}\theta^{\circ} \text { pour WO } \\
\text { obtenu } \\
\text { à partir de }\end{array}$ & \multicolumn{2}{c}{$\begin{array}{c}\Delta \theta \\
\text { Références }\end{array}$} & $\begin{array}{c}\text { mesurée } \\
\text { à partir de }\end{array}$ & $h k l$ \\
$(19)$ & $(20)$ & WN & WR & $(19)$ & $(20)$ & WN & WR & \\
\hline 11,554 & 11,554 & 11,55 & 11,66 & 0 & 0 & 0 & 0 & 002 \\
12,174 & 12,181 & 12,19 & 12,20 & 0,620 & 0,627 & 0,64 & 0,54 & 200 \\
14,302 & 14,307 & 14,29 & 14,55 & 2,748 & 2,753 & 2,74 & 2,69 & 112 \\
16,626 & 16,637 & & & 5,072 & 5,073 & & & 022 \\
16,774 & 16,779 & 16,71 & 16,75 & 5,220 & 5,225 & 5,16 & 5,09 & 202
\end{tabular}

tableau ne constitue donc qu'un élément d'appréciation moyen basé sur l'étude de deux couches parmi d'autres.

Les différents spectres montrent une texture marquée avec les plans (001) orientés parallèlement au support. La comparaison des spectres indique de plus qu'il semble que les échantillons préparés suivant les modes 1 ou 3 présentent une meilleure cristallisation que ceux préparés suivant le mode 2 . Cependant pour toutes les couches, quel que soit le mode de recuit, la cristallisation est d'autant meilleure que l'épaisseur est grande.

3.2.2 Spectroscopie Raman. - Les mesures ont été faites au laboratoire de Physique des Liquides et Electrochimie, Université P. et M. Curie, Paris.

La figure 3a et $\mathrm{a}^{\prime}$ représente un spectre de diffusion Raman obtenu sur une couche de $\mathrm{WO}_{3}$, préparée par recuit dans l'oxygène à $500^{\circ} \mathrm{C}$ d'un film de tungstène réflecteur (WR). Les spectres obtenus à partir de tous les autres échantillons sont absolument semblables.

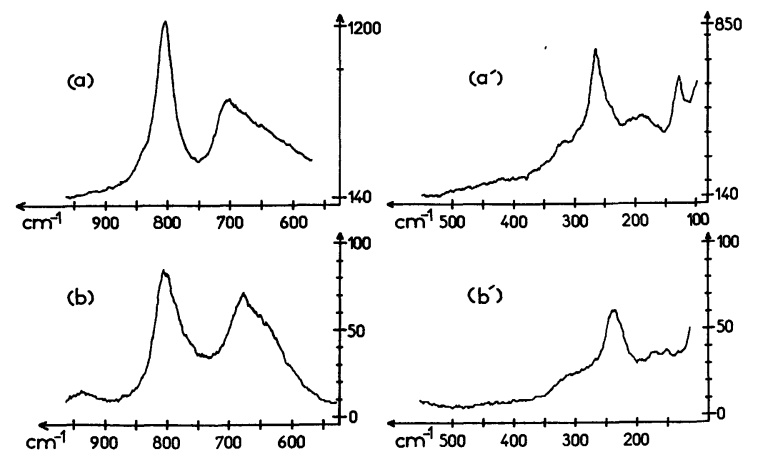

Fig. 3. - Spectre Raman d'un échantillon de $\mathrm{WO}_{3}$ préparé par recuit à $500^{\circ} \mathrm{C}$ sous oxygène d'un film de tungstène réflecteur WR ( $a$ et $\left.a^{\prime}\right)$ et d'une poudre de $\mathrm{WO}_{3}$ hexagonal ( $b$ et $b^{\prime}$ ).

[Raman-spectra taken on a $\mathrm{WO}_{3}$ sample prepared by annealing at $500^{\circ} \mathrm{C}$ in an oxygen atmosphere of a reflective tungsten RW film (a and $\mathrm{a}^{\prime}$ ) and on a hexagonal $\mathrm{WO}_{3}$ powder $\left(b\right.$ and $\left.b^{\prime}\right)$.]
Des études récentes $[6,21]$ sur des films de $\mathrm{WO}_{3}$ obtenus par oxydation anodique en milieu sulfurique, ont montré que ce matériau est amorphe lorsqu'il est préparé à basse tension, et cristallise lorsque la tension anodique dépasse $58 \mathrm{~V}$ suivant la modification hexagonale, récemment synthétisée à partir de l'hydrate tungstique $\mathrm{WO}_{3}, 1 / 3 \mathrm{H}_{2} \mathrm{O}$ [22]. Sur la figure $3 b$ et $b^{\prime}$ nous avons tracé le spectre de diffusion Raman de la poudre ainsi synthétisée. D'évidence, ce spectre est différent de celui de notre échantillon (CVD). L'écart entre les deux pics relatifs aux vibrations d'élongation O-W-O (817 et $690 \mathrm{~cm}^{-1}$ pour la modification hexagonale) est significatif, ainsi que la position du deuxième pic $\left(690 \mathrm{~cm}^{-1}\right)$. On peut encore considérer comme significative la position du pic principal de déformation des groupes $\mathrm{O}-\mathrm{W}-\mathrm{O}\left(255 \mathrm{~cm}^{-1}\right.$ pour la modification hexagonale). Cependant, il faut considérer que dans les différentes modifications de $\mathrm{WO}_{3}$, l'environnement en atomes d'oxygène d'un atome de tungstène déterminé n'est pas fondamentalement différent ; c'est l'arrangement entre eux, des octaèdres élémentaires, autour de canaux carrés (modification monoclinique) ou hexagonaux (modification hexagonale) qui permet la discrimination; il ne faut donc pas s'étonner d'obtenir des spectres Raman peu différenciés. Signalons de plus l'absence sur les spectres relatifs à nos échantillons du pic localisé aux environs de $950 \mathrm{~cm}^{-1}$, pic qui existe (en spectroscopie Raman et Infrarouge) pour les échantillons de $\mathrm{WO}_{3}$ hydratés (ou colorés en présence d'électrolyte aqueux) préparés par d'autres méthodes [6, 23-27].

Les spectres Raman d'une poudre monoclinique ainsi que ceux de poudre hexagonale récemment synthétisée viennent d'être publiés [28, 29]. Dans le tableau II ont été rassemblés un certain nombre de résultats de spectroscopie Raman. En ce qui concerne l'échantillon hexagonal, l'accord entre les spectres obtenus dans les deux laboratoires est excellent; l'écart entre le spectre de la poudre monoclinique et celui de notre échantillon CVD est donc significatif. Il est à signaler du reste que les spectres des films anodiques hexagonaux ne reproduisent pas non plus les spectres de poudre [6], mais sont très voisins de ceux que nous obtenons sur notre échantillon CVD. Ceci peut être expliqué de la façon suivante : les identifications cristallographiques après diffraction d'électrons [6] ou de rayons $\mathrm{X}$ (présent travail) sont essentiellement une cartographie de l'arrangement des cations ; la spectroscopie Raman est plus directement sensible aux liaisons W-O. Dans la modification hexagonale, toutes les liaisons tungstène-oxygène sont de longueurs égales $(1,9 \AA)$; dans la modification monoclinique, trois liaisons sont de longueur $1,8 \AA$, et trois autres, $2,1 \AA$. Il est donc logique de penser que dans les films hexagonaux comme dans les présents films monocliniques, une certaine dispersion existe en ce 
Tableau II. - Position des pics de spectroscopie Raman (en $\left.\mathrm{cm}^{-1}\right)$.

[Positions of the peaks of the Raman spectroscopy $\left(\mathrm{cm}^{-1}\right)$.]

\begin{tabular}{lccccccccc}
\hline Sur nos échantillons CVD & \multicolumn{2}{c}{$808-806$} & $707-690$ & 320 & 265 & $191-186$ & \multicolumn{2}{c}{129} \\
\hline $\mathrm{WO}_{3}$ monoclinique [30] & 808 & 719 & 330 & 275 & & 133 & 93 \\
\hline $\mathrm{WO}_{3}$ monoclinique [28-29] & 807 & 715 & & 327 & 273 & 198 & & 134 & \\
\hline $\mathrm{WO}_{3}$ hexagonal [28-29] & 817 & & $690-645$ & 320 & 253 & 162 & 108 \\
\hline $\mathrm{WO}_{3}$ hexagonal [21] & 816 & & 686 & 318 & 248 & 160 & \\
\hline
\end{tabular}

qui concerne les distances W-O, traduite par la valeur intermédiaire de $701 \mathrm{~cm}^{-1}$ pour le second pic relatif à la vibration d'élongation $\mathrm{O}-\mathrm{W}-\mathrm{O}$. Ce désordre en oxygène pourrait être dû à un certain écart à la stœchiométrie, ce que nous allons vérifier par RBS.

3.3 Composition. - Les mesures de la stœchiométrie des couches ont été faites par rétrodiffusion Rutherford de particules alpha au Département de Physique des Matériaux de l'Université Claude Bernard Lyon I. Les différents spectres obtenus ont montré que la composition des couches dépendaient des conditions de préparation. Pour les couches préparées à $500^{\circ} \mathrm{C}$ dans l'air, il est apparu une inhomogénéité en volume, avec une surstœchiométrie en oxygène en surface et une surstæchiométrie en tungstène du côté du support, comme le montre la figure 4 relative à une couche obtenue par oxydation d'un film de tungstène noir (avant dernière ligne

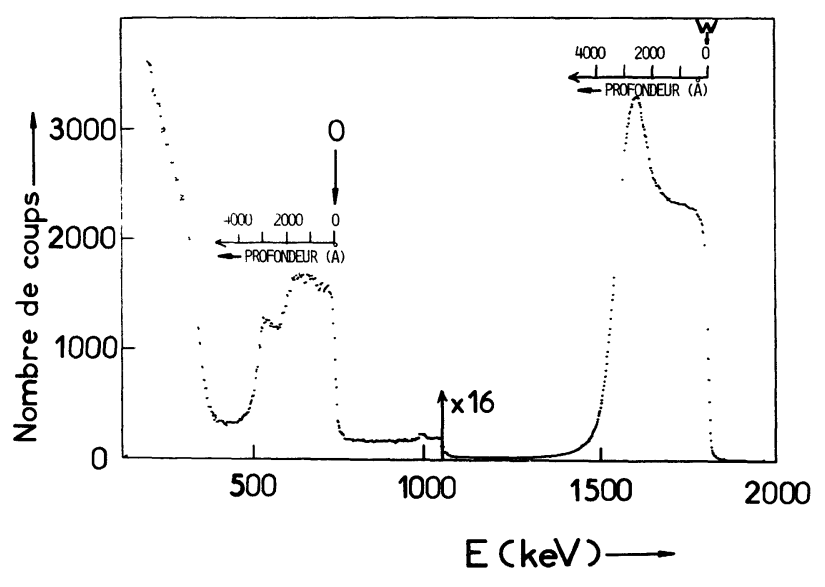

Fig. 4. - Spectre de rétrodiffusion Rutherford pour une couche de tungstène noir déposée sur un substrat de carbone et recuite à $500^{\circ} \mathrm{C}$ dans l'air.

[RBS spectra for a black tungsten BW film prepared on a carbon substrate and annealed at $500{ }^{\circ} \mathrm{C}$ in air.]

Tableau III. - Composition des différentes couches préparées.

[Composition of the various studied samples.]

\begin{tabular}{|c|c|c|c|c|c|c|}
\hline $\begin{array}{c}\text { Matériau } \\
\text { initial }\end{array}$ & Recuits & & W/O & $\begin{array}{c}\text { Stœchiométrie } \\
\text { globale }\end{array}$ & Formule & Observations \\
\hline \multirow{3}{*}{$\begin{array}{l}\text { Tungstène } \\
\text { reflecteur }\end{array}$} & $600^{\circ} \mathrm{C}$ dans l'air & & $\begin{array}{l}0,31 \\
0,28\end{array}$ & $\begin{array}{l}0,31 \\
0,28\end{array}$ & $\begin{array}{l}\mathrm{WO}_{3,22} \\
\mathrm{WO}_{3,57}\end{array}$ & homogène \\
\hline & $500^{\circ} \mathrm{C}$ dans l'air & $\begin{array}{c}\text { en surface } \\
0,28 \\
0,29\end{array}$ & $\begin{array}{c}\text { vers le support } \\
0,38 \\
0,32\end{array}$ & $\begin{array}{l}0,33 \\
0,35\end{array}$ & $\begin{array}{l}\mathrm{WO}_{3} \\
\mathrm{WO}_{2,85}\end{array}$ & inhomogène \\
\hline & $500^{\circ} \mathrm{C}$ dans l'oxygène & & 0,33 & 0,33 & $\mathrm{WO}_{3}$ & homogène \\
\hline \multirow{3}{*}{$\begin{array}{l}\text { Tungstène } \\
\text { noir }\end{array}$} & $600^{\circ} \mathrm{C}$ dans l'air & & 0,31 & 0,31 & $\mathrm{WO}_{3,22}$ & homogène \\
\hline & $500^{\circ} \mathrm{C}$ dans l'air & $\begin{array}{c}\text { en surface } \\
0,29\end{array}$ & $\begin{array}{c}\text { vers le support } \\
0,51\end{array}$ & 0,36 & $\mathrm{WO}_{2,77}$ & inhomogène \\
\hline & $500^{\circ} \mathrm{C}$ dans l'oxygène & & 0,32 & 0,32 & $\mathrm{WO}_{3,12}$ & homogène \\
\hline
\end{tabular}


du tableau III). La stœchiométrie globale montrerait une légère soustœchiométrie en oxygène.

Pour les autres espèces d'échantillons recuits à $600^{\circ} \mathrm{C}$ dans l'air ou à $500^{\circ} \mathrm{C}$ mais en présence d'oxygène, les spectres montrent une bonne homogénéité en volume (par exemple la figure 5 correspond aux résultats de la dernière ligne du tableau III) avec une surstœchiométrie en oxygène.

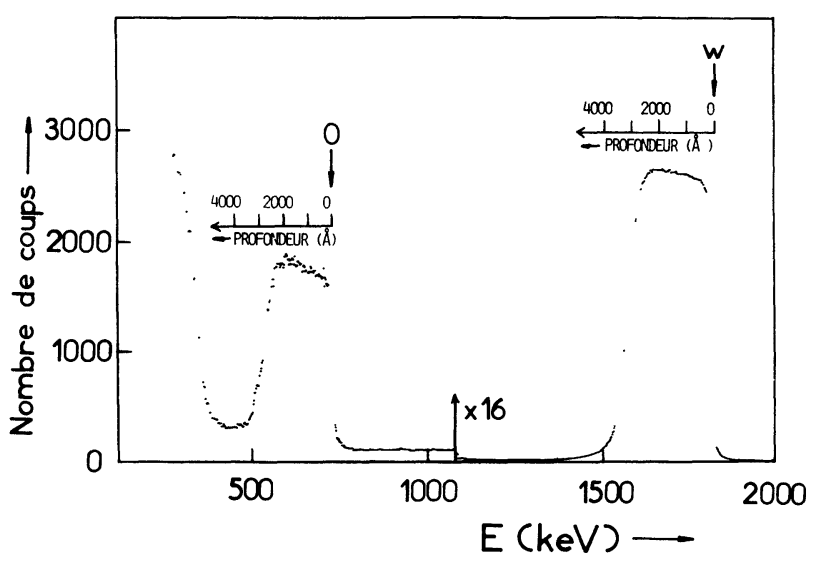

Fig. 5. - Spectre de rétrodiffusion Rutherford pour une couche de tungstène noir déposée sur un substrat de carbone et recuite à $500^{\circ} \mathrm{C}$ dans l'oxygène.

[RBS spectra taken on a black tungsten $\mathrm{BW}$ film prepared on carbon substrate and annealed at $500^{\circ} \mathrm{C}$ in oxygen.]

Dans le tableau III ont été rassemblés les résultats obtenus sur les différentes espèces d'échantillons préparés. Peu de travaux relatifs à des mesures de RBS sur $\mathrm{WO}_{3}$ en couches minces existent, cependant les résultats publiés montrent l'influence des méthodes de préparation sur la composition des matériaux obtenus [31-33].

3.4 Propriétés optiques. - Après recuit les couches sont transparentes tout en présentant une légère coloration jaune. Sur la figure 6 sont représentés les spectres de réflexion et transmission obtenus sur une couche de tungstène réflecteur recuite à $600^{\circ} \mathrm{C}$ dans l'air. La présence des franges d'interférences permet de déterminer les constantes optiques (indices, coefficient d'absorption $\alpha, \ldots$ ) puis d'en déduire à partir de la relation de Tauc $(\alpha E)^{1 / 2}=$ $B\left(E-E_{\mathrm{g}}\right)$ le gap optique $E_{\mathrm{g}}$. L'ensemble des couches a fourni des spectres semblables. Le détail concernant la détermination des caractéristiques optiques a été décrit précédemment $[13,34]$. Il n'a pas été possible de mettre en évidence pour les variations des indices avec l'épaisseur une règle commune aux trois modes de recuit. Ceci peut être attribué à l'effet conjugué de plusieurs paramètres (cristallisation, rugosité, taille des cristallites, lacunes, défauts ponctuels, stœchiométrie, etc.) qui peuvent affecter différemment la valeur des indices.

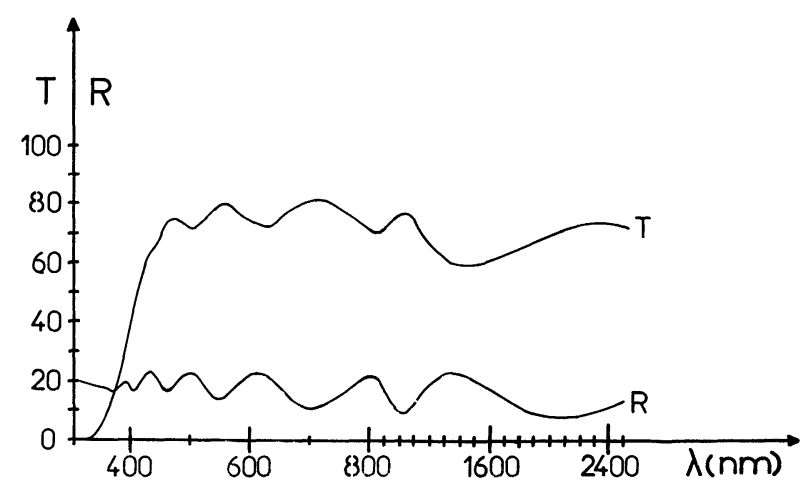

Fig. 6. - Spectre de réflexion et transmission pour une couche de tungstène réflecteur recuite à $600^{\circ} \mathrm{C}$ dans l'air (attention au changement d'échelle en abscisse).

[Typical reflection and transmission spectra for a reflective tungsten film annealed at $600^{\circ} \mathrm{C}$ in air (please pay attention to the scale changement).]

Cependant pour chaque échantillon l'indice de réfraction, $n$, croît d'une manière monotone avec l'énergie du photon. Nous pouvons indiquer que, pour $\lambda=0,5 \mu \mathrm{m}(2,5 \mathrm{eV})$ la valeur de $n$ est comprise entre 1,9 et 2,6 . Ceci est en bon accord avec celles fournies par la littérature : $2,8[1]-1,95[5]$ pour $\mathrm{WO}_{3}$ amorphe et $1,8[6]-2,5[35]$ pour les poly et monocristaux. Les variations de l'indice d'extinction, $k$, avec l'énergie du photon sont semblables pour tous les échantillons. La valeur de $k$ est sensiblement nulle entre 1,5 et $3 \mathrm{eV}$ et croît de chaque côté de cet intervalle d'énergie sans jamais excéder la valeur de 0,3 .

Le gap optique diminue lorsque l'épaisseur de couches augmentent pour tous les types d'échantillons c'est-à-dire quand la cristallisation augmente. Sa valeur varie depuis $3,2-3 \mathrm{eV}$ pour les couches les plus minces jusqu'à 2,9-2,5 eV pour les plus épaisses. Ces résultats sont en accord avec ceux publiés pour les couches de $\mathrm{WO}_{3}$ amorphes $[1,36,37]$ et polycristallines [37, 38]. Signalons que pour des films anodiques polycristallins, le seuil d'absorption déduit de la courbe de variation du coefficient d'absorption avec l'énergie prend une valeur plus élevée, proche de celle des bronzes hexagonaux [6].

3.5 PropriétÉS ÉLECTROCHROMES. - La figure 7a montre les spectres optiques de transmission d'une couche de $\mathrm{WO}_{3}$ préparée par oxydation de tungstène noir à $600^{\circ} \mathrm{C}$ dans l'air pour différentes valeurs de tension appliquée entre l'électrode de travail et l'électrode de platine. Les courbes pleines sont relatives aux spectres obtenus lorsque la tension est appliquée en permanence pendant l'enregistrement (15 min environ), celles en tirets ont été tracées après que la tension ait été appliquée pendant seulement une minute avant le début de l'enregistrement. On observe une variation graduelle et réversi- 


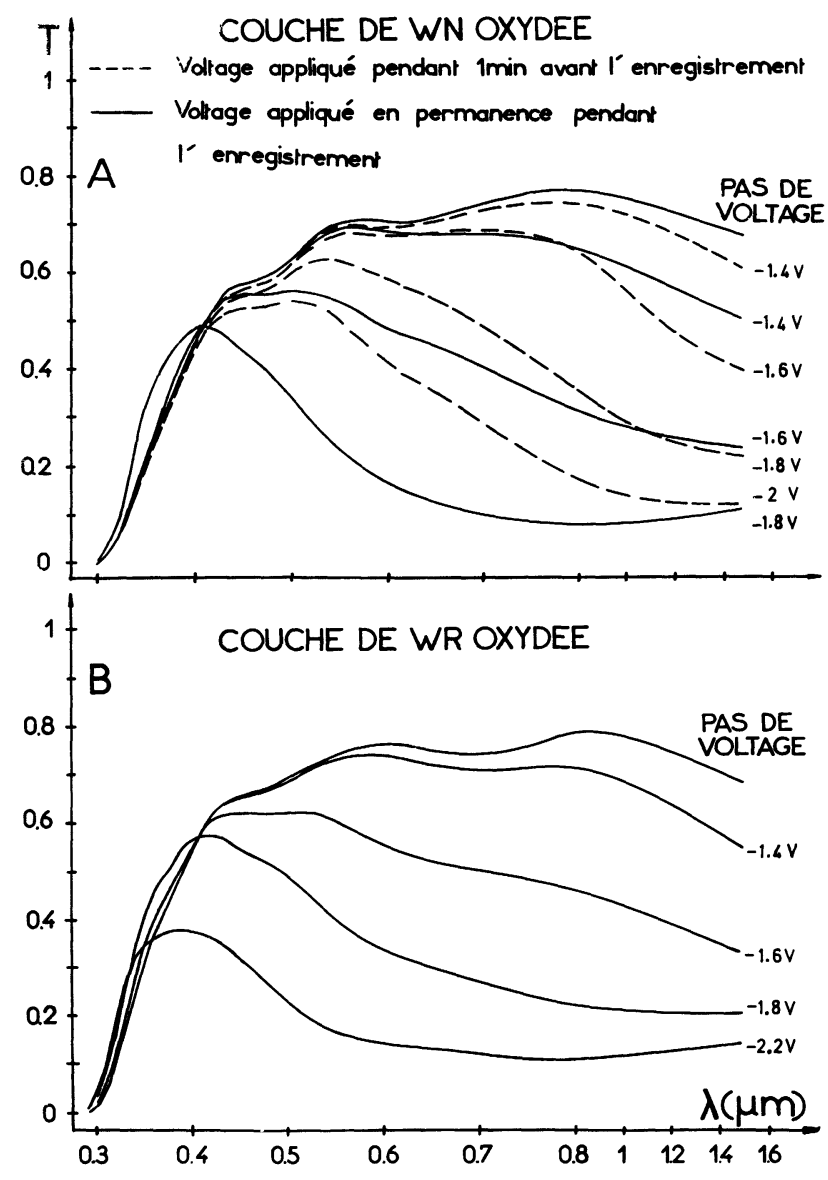

Fig. 7. - Spectres optiques de transmission de couches obtenues à $600^{\circ} \mathrm{C}$ dans l'air en fonction du potentiel appliqué. A) Couche de $1400 \AA$ obtenue par oxydation de tungstène noir. B) Couche de $660 \AA$ obtenue par oxydation de tungstène réflecteur.

[Transmission spectra for two films annealed at $600{ }^{\circ} \mathrm{C}$ in air versus applied potential. A) A $1400 \AA$ thick film obtained by oxidation of a black tungsten layer. B) A $660 \AA$ thick film obtained by oxidation of a reflective tungsten layer.]

ble de la transmission entre de larges limites, spécialement dans le domaine du proche infrarouge. Il faut aussi signaler l'apparition d'une décoloration quand seul l'effet mémoire est considéré (tension de coloration non appliquée en permanence). La figure $7 \mathrm{~b}$ montre les phénomènes observés avec une couche obtenue par oxydation de tungstène réflecteur à
$600{ }^{\circ} \mathrm{C}$ dans l'air lorsque la tension était appliquée en permanence. Les courbes ont des allures semblables aux précédentes. Il faut observer cependant que les tensions appliquées sont légèrement plus élevées.

Pour tous les échantillons étudiés, quel que soit leur mode de recuit c'est-à-dire leur stœchiométrie, les mêmes phénomènes électrochromes apparaissent. Ceci est un résultat très intéressant car même les couches présentant une déficience en tungstène sont électrochromes. Jusqu'alors le phénomène avait été montré dans des échantillons de $\mathrm{WO}_{3}$ présentant une déficience d'oxygène $[1,39,40]$ ou stœchiométriques [21]. Une étude détaillée des performances électrochromes de nos échantillons est en cours, les premiers résultats ont été publiés [41]. Elle montre la grande complexité des phénomènes ainsi que leur dépendance vis-à-vis des divers paramètres de préparation et des différents traitements ultérieurs.

\section{Conclusion.}

Nous avons présenté les résultats concernant la préparation, la structure et la composition de couches minces d'oxyde de tungstène présentant des effets électrochromes en vue d'application dans des systèmes de contrôle de transfert d'énergie lumineuse ou d'affichage électro-optique. Les échantillons ont été préparés par oxydation à haute température de composés de tungstène obtenus par CVD. Tous les matériaux produits sont polycristallins et présentent une composition voisine de la stœchiométrie avec plutôt un excès d'oxygène. Le phénomène électrochrome est présent dans tous les échantillons étudiés ce qui représente un résultat intéressant. Une étude, en cours, des performances électrochromes montre des résultats encourageants vers une possibilité d'applications de ces matériaux dans des afficheurs et aussi dans l'industrie du verre : automobile (rétroviseurs anti-éblouissement) et habitat («smart windows»).

\section{Remerciements.}

Les auteurs remercient le Professeur J. C. Manifacier pour la préparation des substrats de $\mathrm{SnO}_{2}$ et Monsieur L. Martin pour les enregistrements des spectres optiques.

\section{Bibliographie}

[1] Deb, S. K., Philos. Mag. 27 (1973) 801.

[2] Faughnan, B. W., Crandall, R. et Heyman, P. M., RCA Rev. 36 (1975) 177.

[3] Arnoldussen, T. C., J. Electrochem. Soc. 123 (1976) 527.
[4] Deneuville, A., Gerard, P. et Billat, R., Thin Solid Films 70 (1980) 203-223.

[5] Bohnke, O., Thèse Besançon, 1984.

[6] Falaras, P., Thèse Paris, 1986.

[7] LAMPERT, C. M., Solar Energy Materials 11 (1984) 127. 
[8] Svensson, F. S. E. M. et Granqvist, C. G., SPIE Meeting San Diego (August 1984).

[9] Schuster, A. P., Nguyen, D. et Caporaletti, O., Solar Energy Materials 13 (1986) 153.

[10] Matsuhiro, K. et Masuda, Y., SID Digest 10 (1979) 78.

[11] Matsuhiro, K. et Masuda, Y., Proc. SID 21 (1980) 101.

[12] Yous, B., Thèse Montpellier, 1985.

[13] Davazoglou, D. et Donnadieu, A., Thin Solid Films 147 (1987) 131.

[14] Donnadieu, A. et Davazoglou, D., 3rd SPIE Symp. Innsbruck (14-18 April 1986) 653 (1986) 36.

[15] ChaOui, A., Thèse Montpellier, 1984.

[16] Davazoglou, D., Colloque International sur la Science des Matériaux pour l'énergie, Trieste, sept. 1986.

[17] Benard, J., L'oxydation des métaux (Gauthier-Villars, Paris) 1964, t. II, p. 252.

[18] Nachtigal, E., Z. Metall 43 (1952) 23.

[19] Loopstra, B. O. et Rietveld, H. M., Acta Cryst. B 25 (1969) 1420.

[20] Salje, E., Viswassathan, L., Acta Cryst. A 31 (1975) 356.

[21] Falaras, P., Froelicher, M., Froment, M. et Hugot-Legoff, A., J. Microsc. Spectrosc. Electron 9 (1984) 39.

[22] Gerand, G., Nowogrocki, G., Guenot, J. et FiglarZ, M., J. Solid State Chem. 29 (1979) 429.

[23] Salje, E., Acta Cryst. A 31 (1975) 360.

[24] Ramans, G. M., Gabrusenoks, J. V. et Veispals, A. A., Phys. Status Solidi A 74 (1982) K41.

[25] Mercier, R., Bohnke, O., Bohnke, C., Robert, G., Carquille, B. et Mercier, M. F., Mater. Res. Bull. 18 (1983) 1.
[26] Le Bihan, R. et Grandet, G., Le Vide, Les Couches Minces 222 (1984) 213.

[27] Ramans, G. M., Gabrusenoks, J. V., Lusis, A. R. et Patmalnieks, A. A., J. Non Cryst. Solids 90 (1987) 637.

[28] Daniel, M. F., Thèse Bordeaux, novembre 1986.

[29] Daniel, M. F., Desbat, B., Lassegues, J. C., Gerand, B. and Figlarz, M., J. Solid State Chem. 67 (1987) 3688.

[30] Salje, E., Acta Cryst. A 32 (1976) 233.

[31] Ziegler, J. F. et Baglin, E. E., J. Appl. Phys. 45 (1974) 1888.

[32] Mc Intyre, J. D. E., Basu, S., Peck, W. F. Jr, Brown, W. L. and Augustyniak, W. M., Phys. Rev. B 25 (1982) 7242.

[33] Engelmann, C., Loeuillet, M. et Bohnke, O., $J$. Trace Microprobe Techniques 4 (1 \& 2) (1986) 93.

[34] Davazoglou, D., Leveque, G. and Donnadieu, A., J. Applied Phys., soumis pour publication.

[35] Swada, S. et Danielson, C. G., Phys. Rev. 113 (1969) 1008.

[36] Nakamura, A. et Samada, Y., Appl. Phys. 24 (1981) 55.

[37] Diquarto, F., Di Paola, A., Piazza, S. et SunSERI, C., Solar Energ. Mat. 11 (1985) 419.

[38] Butler, M. A., MAshby, R. D. et QuinN, R. K., Solid State Commun. 19 (1976) 1011.

[39] Deneuville, A. et Gerard, P., J. Electron. Mater. 7 (1978) 539.

[40] Kamimori, T., NAGAI, J. and Mizuhashi, M., SPIE Innsbruck 653 (1986) 2.

[41] Davazoglou, D., Donnadieu, A. et Bohnke, O., Solar Energ. Mat. 16 (1987) 55. 